\title{
Stroke care: Leaps in addressing the shortfall with some Angels to the rescue
}

One in six people is likely to have a stroke in their lifetime, and the condition remains a leading cause of death and adult disability worldwide. For years, stroke patients were treated with 'benign neglect' based on the premise that the stroke victim's fate was sealed soon after onset and nothing much could be done apart from calling in the rehabilitation team a few days later if the patient survived. However, there have been four major quantum leaps in the management of acute stroke over the past decade or two that have resulted in improved outcomes.

The stroke unit model of care, embracing the concept of a multidisciplinary team working synchronously using standard operating procedures and pathways to maintain homeostasis, initiate appropriate early rehabilitation and prevent complications, has been shown in numerous studies worldwide to be effective in improving outcome by reducing both mortality and morbidity. This model of care was shown to be effective in substantially reducing mortality in a small under-resourced community hospital in Western Cape Province, South Africa (SA). ${ }^{[1]}$

Reperfusion therapy in the form of intravenous thrombolysis with tissue plasminogen activator ( $\mathrm{tPA}$ ) was the next major leap in the management of acute ischaemic stroke. Despite occasional sporadic dissenting voices concerned with the very real risks of this treatment modality, intravenous thrombolysis has been recommended as an option for suitable patients by the World Stroke Organization, the European Stroke Organisation and most individual country stroke societies including the South African Stroke Society. ${ }^{[2]}$ Although intravenous thrombolysis may be associated with bleeding and worsening of the stroke, and even death in a small percentage of patients, the net benefit exceeds the risk in carefully selected patients, resulting in an increased number of patients whose stroke symptoms are reversed or lessened by the treatment. The indications for, contraindications to and potential benefit and harm of intravenous thrombolysis have been well elucidated in the various guidelines including the most recent American Heart Association/American Stroke Association guideline published in 2018. ${ }^{[3]}$ However, the narrow therapeutic window from symptom onset to treatment with a thrombolytic agent is one of the major limitations hindering its widespread use in clinical practice.

The next leap forward was the advent of intra-arterial mechanical thrombectomy (MTB) for acute ischaemic stroke within 6 hours of symptom onset, shown in studies to result in the most striking and dramatic improvements in outcomes in selected patients with proximal cerebral artery occlusions. ${ }^{[4-8]}$ However, the procedure requires highly skilled trained interventionalists working in highvolume centres to achieve good results with low complication rates (not insignificant even in good hands). Patient selection, operator skill and timing of MTB are all key determinants of outcome. At present in SA this procedure is limited to one or two centres in the large metropoles.

More recently, an exciting leap at the frontier of stroke management has been the development of advanced radiological software for use on computed tomography (CT) or magnetic resonance imaging providing automated perfusion studies (e.g. RAPID, a validated software that has been used in clinical trials). When executed consistently and interpreted correctly, such automated CT perfusion studies can identify appropriate candidates for thrombectomy. Such software is becoming an important decision-making tool, because it can rapidly identify those patients who have salvageable brain tissue and can benefit from thrombectomy. ${ }^{[9,10]}$ Individuals can be differentiated into those with or without salvageable brain tissue, or for whom the benefits of thrombectomy outweigh the risks. Recent trials have extended the treatment window substantially by showing long-lasting benefit of thrombectomy in a subset of ischaemic stroke patients who present within 24 hours of symptom onset. ${ }^{[11,12]}$ Given the inherent risk of both reperfusion modalities, compliance with recommended guidelines has become all the more important in order to maximise the potential benefit and reduce the associated risks of this treatment.

The pervading medical litigious environment in SA has led to an increasing number of malpractice lawsuits, including those in which hospitals and doctors are being sued for not acting with urgency and offering the option of reperfusion therapy to patients who arrive in the EU within the required time window for reperfusion. Stroke treatment is very much in the cross hairs of malpractice lawyers, and failure to act in accordance with our own guideline, and every other published guideline, including failure to discuss the option of reperfusion in suitably selected patients, is now being used as the basis for the lawsuits. This situation makes the study by Mandizvidza et al. ${ }^{[13]}$ published in this issue of $S A M J$ all the more relevant, as it is going to be increasingly difficult to defend future claims, particularly as large public hospitals such as Groote Schuur, Tygerberg, Livingstone and Johannesburg General have been able to administer such treatment to suitable patients with acute ischaemic stroke for some time now.

Fortunately in the past 2 years there has been enormous progress, largely as a result of the Angels Initiative (an industry-sponsored initiative that has been endorsed by the World Stroke Organization, the European Stroke Organisation and the South African Stroke Society) that has been responsible for on-site training in both public and private hospitals in all aspects of stroke care (with protocols based on international guideline recommendations and vetted by local stroke experts). Training has included on-site simulation testing in order to streamline reperfusion treatment for suitable patients with acute ischaemic stroke and reduce 'door-to-needle' times. Sadly, many SA hospitals are not up to scratch with treating their stroke patients and are now increasingly likely to be vulnerable to litigation. Although the study by Mandizvidza et al. ${ }^{[13]}$ was undertaken in a sample of hospitals in the Cape Metro Health District, it almost certainly reflects the status quo elsewhere in the country, where conditions are often much worse. In hospitals that do not have the resources necessary for reperfusion therapy, treating stroke patients urgently without delay to maintain homeostasis and prevent complications and initiate secondary prevention adopting the stroke unit model of care should by now be possible at all levels of care, regardless of whether or not the patient is a candidate for any type of reperfusion therapy.

Given the considerable stroke burden in SA, an evaluation of the barriers to acute stroke care in the public health care sector (also present in many private hospitals) is timely in response to the failure of many hospitals with the capacity to react appropriately in managing such patients. The SA stroke guideline, ${ }^{[2]}$ published in SAMJ 10 years ago, was tailored to the limited resources available at the different levels of the public health care sector. Although the Angels Initiative has done much to address the gaps in stroke care in 
many hospitals throughout the country, it remains crucial to identify and address the remaining shortfalls and barriers to efficient stroke care in our setting.

\section{Alan Bryer}

Division of Neurology, Department of Medicine, Groote Schuur Hospital and Faculty of Health Sciences, University of Cape Town, South Africa

alan.bryer@uct.ac.za

1. De Villiers L, Kalula SZ, Burch VC. Does multidisciplinary stroke care improve outcome in secondary-level hospital in South Africa? Int J Stroke 2009;4(2):89-93. https://doi.org/10.1111/j.1747 4949.2009.00254.x

2. Bryer A, Connor M, Haug P, et al. South African guideline for management of ischaemic stroke and transient ischaemic attack 2010: A guideline from the South African Stroke Society (SASS) and the SASS Writing Committee. S Afr Med J 2010;100(11 Pt 2):747-778.

3. Powers WJ, Rabinstein AA, Ackerson T, et al. 2018 Guidelines for the Early Management of Patients with Acute Ischemic Stroke: A guideline for healthcare professionals from the American Heart Association/American Stroke Association. Stroke 2018;49(3):e46-el10. https://doi.org/10.1161/ Association/American

4. Berkhemer OA, Fransen PSS, Beumer D, van den Berg LA, Lingsma HF, Yoo AJ. A randomized trial of intraarterial treatment for acute ischemic stroke. N Engl J Med 2015;372:11-20. https://doi. trial of intraarterial treatment
org/10.1056/NEJMoa 1411587
5. Goyal M, Demchuk AM, Menon BK, et al. Randomized assessment of rapid endovascular treatment of ischemic stroke. N Engl J Med 2015;372(11):1019-1030. https://doi.org/10.1056/NEJMoal414905 6. Campbell BCV, Mitchell PJ, Kleinig TJ, Dewey HM, Churilov L, Yassi N. Endovascular therapy for 6. Campbell BCV, Mitchell Pf, Kleinig TJ, Dewey HM, Churilov L, Yassi N. Endovascular therapy for
ischemic stroke with perfusion-imaging selection. N Engl J Med 2015;372:1009-1018. https://doi. ischemic stroke with perfusion
org/10.1056/NEJMoa 1414792

7. Saver JL, Goyal M, Bonafe A, Diener H-C, Levy EI, Pereira VM. Stent-retriever thrombectomy after intravenous t-PA vs. t-PA alone in stroke. N Engl J Med 2015;372(24):2285-2295. https://doi. org/10.1056/NEJMoal415061

8. Young-Saver DF, Gornbein J, Starkman S, Saver JL. Magnitude of benefit of combined endovascular thrombectomy and intravenous fibrinolysis in large vessel occlusion ischemic stroke. Stroke 2019;50(9):2433-2440. https://doi.org/10.1161/STROKEAHA.118.023120

9. Wing SC, Markus HS. Interpreting CT perfusion in stroke. Pract Neurol 2019;19(2):136-142. https:// doi.org/10.1136/practneurol-2018-001917

10. Lansberg MG, Lee J, Christensen S, Straka M, de Silva DA, Mlynash M. RAPID automated patient selection for reperfusion therapy. Stroke 2011;42(6):1608-1614. https://doi.org/10.1161/ STROKEAHA.110.609008

11. Albers GW, Marks MP, Kemp S, et al. Thrombectomy for stroke at 6 to 16 hours with selection by perfusion imaging. N Engl J Med 2018;378(8):708-718. https://doi.org/10.1056/NEJMoa1713973

2. Nogueira RG, Jadhav AP, Haussen DC, et al. Thrombectomy 6 to 24 hours after stroke with a mismatch between deficit and infarct. N Engl J Med 2018;378(1):11-21. https://doi.org/10.1056/NEJMoa1706442
b. 13. Mandizvidza V, London L, Bryer A. Shortfall in stroke care: A study of ischaemic stroke care practices in a South African metropole. S Afr Med J 2021;111(3):215-219. https://doi.org/10.7196/SAMJ.2021. v111i3.15104

S Afr Med J 2021;111(3):196-197. https://doi.org/10.7196/SAMJ.2021.v111i3.15103 